\title{
A study of flow structure of bichromatic waves through PIV analysis ${ }^{\dagger}$
}

\author{
Hyo-Jae Jo ${ }^{1}$, Seung-Jae Lee ${ }^{1 *}$ and Je-Eun $\mathrm{Choi}^{2}$ \\ ${ }^{1}$ Division of Naval Architecture and Ocean Systems Engineering, Korea Maritime University, Busan 606-791, Korea \\ ${ }^{2}$ Department of Mechanical Engineering, Nihon University, Tokyo, Japan
}

(Manuscript Received December 27, 2011; Revised January 22, 2012; Accepted February 3, 2012)

\begin{abstract}
An experimental study was carried out in order to understand the kinematics of bichromatic waves. Bichromatic waves are generated in a two-dimensional wave tank, and measured by panorama PIV technique, which allows the flow fields to be captured with respect to a spatial coordinate system. We compared wave profiles and velocities of wave particles obtained by experiment with theoretical results using Stokes 1st and 2nd order waves. The velocity distribution at wave crest and trough of the highest and lowest point of a bichromatic wave are investigated in this study.
\end{abstract}

Keywords: Bichromatic waves, Panorama PIV, Second-order Stokes waves.

\section{Introduction}

Korea has recently been carrying out a variety of research and experiments at various locations, to take advantage of the oceans surrounding the Korean peninsula. Research on wave-making tests in wave basins has brought many methods for analyzing topics of linear and nonlinear irregular waves. Hudspeth and Sulisz (1991) carried out wave-making tests of monochromatic waves, considering 2nd order Stokes waves. Moubayed and Williams (1994), and Schäffer (1996) carried out experiments on bichromatic waves. Recent experiments on wave-making addressed measuring wave height along different locations on a wave basin, simulating the Schrödinger equation, and numerically analyzing bichromatic waves. Bichromatic waves are different from those found in a real sea state. However, research on bichromatic waves can explain interactions of wave profiles, and the flow

\footnotetext{
$\dagger$ This manuscript is based on a presentation on 2005 Fall conference of Korean Society of Offshore Engineers.

${ }^{*}$ Corresponding author. Tel.: +82-51-410-4309, Fax.: +82-51-405-8305

E-mail address:slee@hhu.ac.kr

Copyright $\odot$ KSOE 2012.
}

structure of each component wave.

For measuring flow field, Laser Doppler Velocimetry (LDF) gives comparatively high accuracy of measurement of velocity of flow particle (Skjelbreia et al., 1991). Kim et al. (1992) used Laser Doppler Anemometry (LDA) for measuring flow field of transient waves. Longridge et al. (1996) also used results from LDA, and then calculated local and convective acceleration of flow particle. However these methods only allow measurement of velocity at a single fixed location. Swan et al. (2002) carried out a number of experiments at different locations in order to calculate the velocity field on a spatial coordinate system. Hence it is recommended to use PIV (Particle Image Velocimetry) for the calculation of velocity field in a spatial coordinate system by single experiment with minimum error (Sung and Doh, 2004). Chang and Liu (1998) showed that the PIV method can measure velocity and acceleration of water particles in wavebreaking phenomena with better accuracy. Jacobsen et al. (1997) applied the PIV method to the standing wave case, and Jensen et al. (2001) estimated time derivatives of the velocity of water particles using 
two cameras and a PIV system.

Recent research on analysis of flow field utilizes PIV for quantitative measurement of complex flow field, since the PIV system in particular is considered to be the best measurement system for the nonlinear flow structure of waves.

In this study, panorama PIV techniques are applied for analysis of flow structure of a bichromatic wave which consists of two regular waves with different frequencies, and is generated in a 2-D wave basin. When two regular waves are combined, nonlinear interaction effects are observed, so the characteristics of flow structure need to be investigated. Theoretical calculations using Stokes wave theory based on potential theory are compared to the results from experiments, and then the applicability of theoretical calculation and characteristics of a flow field is investigated.

\section{Formulation}

Under the assumption of inviscid fluid, irrotational flow, and negligible surface tension, velocity potential inside the flow satisfies the Laplace equation and Bernoulli equation.

$$
\begin{array}{ll}
\frac{\partial^{2} \phi}{\partial x^{2}}+\frac{\partial^{2} \phi}{\partial z^{2}}=0 \quad\left(\frac{d \phi}{d x}=u,\right. & \left.\frac{d \phi}{d z}=w\right) \\
\frac{p}{\rho}+g z+\frac{1}{2} q^{2}+\frac{\partial \phi}{\partial t}=0 & \left(q^{2}=u^{2}+w^{2}\right)
\end{array}
$$

( $\phi$ : Velocity potential, $x$ : horizontal distance, $z$ : vertical distance, $u$ : horizontal velocity, $w$ : vertical velocity, $p$ : pressure, $\rho$ : density, $t$ : time, $g$ : gravitational acceleration).

The $\mathrm{x}$-direction means the direction of wave propagation, and the z-direction means the vertical downward direction. The boundary conditions are as follows:

$$
\begin{array}{ll}
\frac{\partial \eta}{\partial t}+u \frac{\partial \eta}{\partial x}=\frac{\partial \phi}{\partial z} & (\text { at } z=\eta) \\
g \eta+\frac{1}{2} q^{2}+\frac{\partial \phi}{\partial t}=0 & (\text { at } z=\eta) \\
\frac{\partial \phi}{\partial z}=0 & (\text { at } z=-h)
\end{array}
$$

\section{$2.11^{\text {st }}$ Order Approximation of Stokes Wave}

1) Velocity potential

$$
\begin{gathered}
\phi=a_{1} c_{1} \frac{\cosh k_{1}(h+z)}{\sinh k_{1} h} \sin k_{1}\left(x-c_{1} t\right)+ \\
a_{2} c_{2} \frac{\cosh k_{2}(h+z)}{\sinh k_{2} h} \operatorname{sihk}_{2}\left(x-c_{2} t\right)
\end{gathered}
$$

2) Wave profile

$\eta=a_{1} \cos k_{1}\left(x-c_{1} t\right)+a_{2} \cos k_{2}\left(x-c_{2} t\right)$

$\left(c_{1}^{2}=\frac{g}{k_{1}} \tanh k_{1} h, \quad c_{2}^{2}=\frac{g}{k_{2}} \tanh k_{2} h\left(k_{1}, k_{2}>0\right)\right)$

3) Velocity

$$
\begin{gathered}
u=a_{1} c_{1} k_{1} \frac{\cosh k_{1}(h+z)}{\sinh k_{1} h} \cos k_{1}\left(x-c_{1} t\right)+ \\
a_{2} c_{2} k_{2} \frac{\cosh k_{2}(h+z)}{\sinh k_{2} h} \cos k_{2}\left(x-c_{2} t+\theta\right) \\
w=a_{1} c_{1} k_{1} \frac{\sinh k_{1}(h+z)}{\sinh k_{1} h} \sin k_{1}\left(x-c_{1} t\right)+ \\
a_{2} c_{2} k_{2} \frac{\sinh k_{2}(h+z)}{\sinh k_{2} h} \sin k_{2}\left(x-c_{2} t+\theta\right)
\end{gathered}
$$

\section{$2.11^{\text {st }}$ Order Approximation of Stokes Wave}

$$
\begin{aligned}
& \frac{1}{2} \frac{\partial}{\partial t}(q u)^{2}+\frac{\partial^{2} \phi_{(2)}}{\partial t_{2}}+\frac{\partial \eta_{(1)}}{\partial t} \frac{\partial^{2} \phi_{(1)}}{\partial z \partial t} \\
& +\eta_{(1)} \frac{\partial^{2} \phi_{(1)}}{\partial z \partial t \partial t}-\left[g \frac{\partial \phi_{(1)}}{\partial x} \frac{\partial \eta_{(1)}}{\partial x}-g \frac{\partial \phi_{(2)}}{\partial z}\right. \\
& \left.-g \eta_{(1)} \frac{\partial^{2} \phi_{(1)}}{\partial z^{2}}\right]=0 \quad(\text { at } \quad \mathrm{z}=0) \\
& \left(\frac{\partial^{2} \phi_{(2)}}{\partial t^{2}}+g \frac{\partial \phi_{(2)}}{\partial z}\right)_{z=0}=\left[-\eta_{(1)} \frac{\partial}{\partial z}\left[\frac{\partial^{2} \phi_{(1)}}{\partial t^{2}}+g \frac{\partial \phi_{(1)}}{\partial z}\right]-\right. \\
& \left.\frac{1}{2} \frac{\partial}{\partial t}\left(q_{(1)}\right)^{2}-\frac{\partial \eta_{(1)}}{\partial t} \frac{\partial^{2} \phi_{(1)}}{\partial z \partial t}+g \frac{\partial \phi_{(1)}}{\partial x} \frac{\partial \eta_{(1)}}{\partial x}\right]_{z=0}
\end{aligned}
$$

1) Velocity Potential 


$$
\begin{aligned}
& \phi=a_{1} c_{1} \frac{\cosh k_{1}(h+z)}{\sinh k_{1} h} \sin k_{1}\left(x-c_{1} t\right)+ \\
& a_{2} c_{2} \frac{\cosh k_{2}(h+z)}{\sinh k_{2} h} \sin k_{2}\left(x-c_{2} t+\theta\right)+ \\
& B 21 \cdot \frac{\cosh 2 k_{1}(h+z)}{\sinh 2 k_{1} h} \sin 2 k_{1}\left(x-c_{1} t\right)+ \\
& B 22 \cdot \frac{\cosh 2 k_{2}(h+z)}{\sinh 2 k_{2} h} \sin 2 k_{2}\left(x-c_{2} t+\theta\right)+ \\
& B 23 \cdot \frac{\cosh \left(k_{1}+k_{2}\right)(h+z)}{\sinh \left(k_{1}+k_{2}\right) h} \sin \left[\left(k_{1}+k_{2}\right) x-\left(c_{1} k_{1}+c_{2} k_{2}\right) t+\theta\right]+ \\
& B 24 \cdot \frac{\cosh \left(k_{1}-k_{2}\right)(h+z)}{\sinh \left(k_{1}-k_{2}\right) h} \sin \left[\left(k_{1}-k_{2}\right) x-\left(c_{1} k_{1}-c_{2} k_{2}\right) t-\theta\right] \\
& B 21=\frac{-\frac{3}{2} a_{1}^{2} c_{1}^{3} k_{1}^{3}\left(D 1^{2}-1\right)}{-4 c_{1}^{2} k_{1}^{2} \cdot D 3+2 g k_{1}} \\
& \mathrm{~B} 22=\frac{-\frac{3}{2} a_{2}^{2} c_{2}^{3} k_{2}^{3}\left(D 2^{2}-1\right)}{-4 c_{2}^{2} k_{2}^{2} \cdot D 4+2 g k_{2}} \\
& B 23=\left\{a_{1} a_{2} c_{1} c_{2} k_{1} k_{2}\left(c_{1} k_{1}+c_{2} k_{2}\right)(1-D 1 \cdot D 2)-\right. \\
& \left.\frac{1}{2} a_{1} a_{2} c_{1}^{3} k_{1}^{3}\left(D 1^{2}-1\right)-\frac{1}{2} a_{1} a_{2} c_{2}^{3} k_{2}^{3}\left(D 2^{2}-1\right)\right\} \\
& /\left\{-\left(c_{1} k_{1}+c_{2} k_{2}\right)^{2} \cdot D 5+g\left(k_{1}+k_{2}\right)\right\} \\
& B 24=\left\{a_{1} a_{2} c_{1} c_{2} k_{1} k_{2}\left(c_{1} k_{1}-c_{2} k_{2}\right)(1+D 1 \cdot D 2)-\right. \\
& \left.\frac{1}{2} a_{1} a_{2} c_{1}^{3} k_{1}^{3}\left(D 1^{2}-1\right)+\frac{1}{2} a_{1} a_{2} c_{2}^{3} k_{2}^{3}\left(D 2^{2}-1\right)\right\} \\
& /\left\{-\left(c_{1} k_{1}-c_{2} k_{2}\right)^{2} \cdot D 6+g\left(k_{1}-k_{2}\right)\right\} \\
& D 1=\operatorname{coth} k_{1} h, D 2=\operatorname{coth} k_{2} h, D 3=\operatorname{coth} 2 k_{1} h, \\
& D 4=\operatorname{coth} 2 k_{2} h, D 5=\operatorname{coth}\left(k_{1}+k_{2}\right) h \text {, } \\
& \left.D 6=\operatorname{coth}\left(k_{1}-k_{2}\right) h\right)
\end{aligned}
$$

\section{2) Wave Profile}

$$
\begin{aligned}
& \eta=a_{1} \cos k_{1}\left(x-c_{1} t\right)+a_{2} \cos k_{2}\left(x-c_{2} t+\theta\right)+ \\
& A 21 \llbracket \cos 2 k_{1}\left(x-c_{1} t\right)+A 22 \sqsubset \cos 2 k_{2}\left(x-c_{2} t+\theta\right)+ \\
& A 23 \sqsubset \cos \left[\left(k_{1}+k_{2}\right) x-\left(c_{1} k_{1}+c_{2} k_{2}\right) t+\theta\right]+ \\
& A 24 \llbracket \cos \left[\left(k_{1}-k_{2}\right) x-\left(c_{1} k_{1}-c_{2} k_{2}\right) t-\theta\right]
\end{aligned}
$$$$
A 21=\frac{1}{g}\left[2 c_{1} k_{1} \square B 21 \square D 3+\frac{1}{2} a_{1}^{2} c_{1}^{2} k_{1}^{2}-\frac{1}{4} a_{1}^{2} c_{1}^{2} k_{1}^{2}\left(D 1^{2}-1\right)\right]
$$

$$
\begin{aligned}
A 22= & \frac{1}{g}\left[2 c_{2} k_{2} \square B 22 \square D 4+\frac{1}{2} a_{2}^{2} c_{2}^{2} k_{2}^{2}-\frac{1}{4} a_{2}^{2} c_{2}^{2} k_{2}^{2}\left(D 2^{2}-1\right)\right] \\
A 23= & \frac{1}{g}\left[\left(c_{1} k_{1}+c_{2} k_{2}\right) \square B 23 \square D 5+\frac{1}{2} a_{1} a_{2}\left(c_{1}^{2} k_{1}^{2}+c_{2}^{2} k_{2}^{2}\right)-\right. \\
& \left.\frac{1}{2} a_{1} a_{2} c_{1} c_{2} k_{1} k_{2}(D 1 \square D 2-1)\right] \\
A 24= & \frac{1}{g}\left[\left(c_{1} k_{1}-c_{2} k_{2}\right) \square B 24 \square D 6\right. \\
& +\frac{1}{2} a_{1} a_{2}\left(c_{1}^{2} k_{1}^{2}+c_{2}^{2} k_{2}^{2}\right) \\
& \left.\quad-\frac{1}{2} a_{1} a_{2} c_{1} c_{2} k_{1} k_{2}(D 1 \square D 2+1)\right]
\end{aligned}
$$

\section{3) Velocity}

$$
\begin{aligned}
u= & a_{1} c_{1} k_{1} \frac{\cosh k_{1}(h+z)}{\sinh k_{1} h} \cos k_{1}\left(x-c_{1} t\right) \\
& +a_{2} c_{2} k_{2} \frac{\cosh k_{2}(h+z)}{\sinh k_{2} h} \cos k_{2}\left(x-c_{2} t+\theta\right) \\
& +2 k_{1} B 21 \cdot \frac{\cosh 2 k_{1}(h+z)}{\sinh 2 k_{1} h} \cos 2 k_{1}\left(x-c_{1} t\right) \\
& +2 k_{2} B 22 \cdot \frac{\cosh 2 k_{2}(h+z)}{\sinh 2 k_{2} h} \cos 2 k_{2}\left(x-c_{2} t+\theta\right) \\
& +\left(k_{1}+k_{2}\right) B 23 \cdot \frac{\cosh \left(k_{1}+k_{2}\right)(h+z)}{\sinh \left(k_{1}+k_{2}\right) h} \cos \left[\left(k_{1}+k_{2}\right) x-\left(c_{1} k_{1}+c_{2} k_{2}\right) t+\theta\right] \\
& +\left(k_{1}-k_{2}\right) B 24 \cdot \frac{\cosh \left(k_{1}-k_{2}\right)(h+z)}{\sinh \left(k_{1}-k_{2}\right) h} \cos \left[\left(k_{1}-k_{2}\right) x-\left(c_{1} k_{1}-c_{1} k_{2}\right) t-\theta\right]
\end{aligned}
$$

$$
\begin{aligned}
w= & a_{1} c_{1} k_{1} \frac{\sinh k_{1}(h+z)}{\sinh k_{1} h} \sin k_{1}\left(x-c_{1} t\right) \\
& +a_{2} c_{2} k_{2} \frac{\sinh k_{2}(h+z)}{\sinh k_{2} h} \sin k_{2}\left(x-c_{2} t+\theta\right) \\
& +2 k_{1} B 21 \cdot \frac{\sinh 2 k_{1}(h+z)}{\sinh 2 k_{1} h} \sinh 2 k_{1}\left(x-c_{1} t\right) \\
& +2 k_{2} B 22 \cdot \frac{\sin 2 k_{2}(h+z)}{\sinh 2 k_{2} h} \sin 2 k_{2}\left(x-c_{2} t+\theta\right) \\
& +(11) \quad\left(k_{1}+k_{2}\right) B 23 \cdot \frac{\sinh \left(k_{1}+k_{2}\right)(h+z)}{\sinh \left(k_{1}+k_{2}\right) h} \sin \left[\left(k_{1}+k_{2}\right) x-\left(c_{1} k_{1}+c_{2} k_{2}\right) t+\theta\right] \\
& +\left(k_{1}-k_{2}\right) B 24 \cdot \frac{\sinh \left(k_{1}-k_{2}\right)(h+z)}{\sinh \left(k_{1}-k_{2}\right) h} \sin \left[\left(k_{1}-k_{2}\right) x-\left(c_{1} k_{1}-c_{2} k_{2}\right) t-\theta\right)
\end{aligned}
$$


The above equations can be used to calculate the profile of a bichromatic wave for 1 st and 2 nd order approximation, and velocity of fluid particle along water depth.

\section{Experiments}

\subsection{Wave-making and Measuring Profile}

In this study, bichromatic waves are generated in two ways. The first method is to compose two component waves of different frequencies. We first generate one component wave of slow phase velocity, and then generate the other wave of fast phase velocity, so that the two component waves are superimposed at a particular location. The second method is to synthesize an electronic signal, which is used as the input of a wave-maker, so that bichromatic waves spread out all around the wave basin.

The reason two methods are used to generate bichromatic waves is to investigate how the flow structures differ between the naturally superposed case of pure composition, and the artificially superposed case of forced composition. Fig. 1 shows the electronic signal for a wave-maker required to generate bichromatic waves composed of two regular waves of two frequencies, $\omega_{1}=7.5$, and $\omega_{2}=6$.

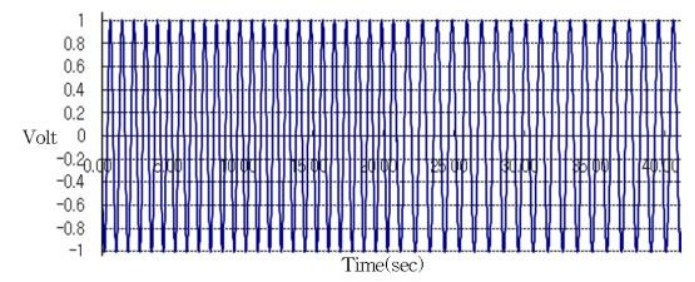

Fig. 1. Input signal for pure composition $\left(\omega_{1}=7.5, \omega_{2}=6\right)$

At a certain location, a bichromatic wave can be observed, and the phase difference of two regular waves can be calculated from the wave profile measured by wave probe. Two cameras are set up where the bichromatic waves are to be observed, and panorama PIV technique is adopted to analyze a wide flow field.

Fig. 2 shows the input signal for a bichromatic wave made by forced composition of regular waves of two frequencies, $\omega_{1}=7.5$ and $\omega_{2}=6$. At a location $15 \mathrm{~m}$ away from the wave-maker, we measure wave profile by wave probe, and look for a wave profile, through analysis of measured wave profile, that has the same phase as in the first method of pure composition. The same panorama PIV technique is applied to the bichromatic wave at a phase we have found, in order to measure the flow field.

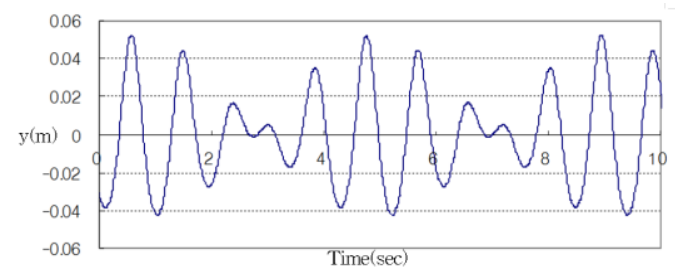

Fig. 2. Input signal for forced composition $\left(\omega_{1}=7.5, \omega_{2}=6\right)$

The experiments are carried out in a 2-D wave basin that has a length of $25 \mathrm{~m}$, breadth of $1 \mathrm{~m}$, and water depth of $0.8 \mathrm{~m}$. The wave maker is of piston type. The profile of a bichromatic wave is measured at a location $15 \mathrm{~m}$ away from the wave-maker, using a capacitive wave probe. Two wave frequencies $\left(\omega_{1}=7.5, \omega_{2}=6\right)$ are selected, by considering the relation between length of wave basin and phase, in order to obtain the most visible bichromatic wave.

\subsection{Panorama PIV System}

The PIV system for measuring particle velocity is configured as shown in Fig. 3:

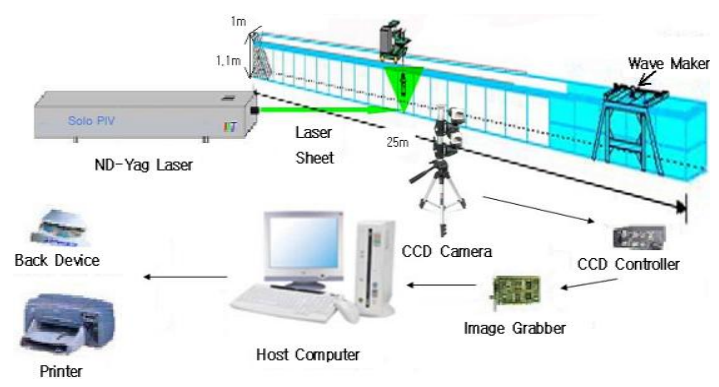

Fig. 2. Panorama-PIV measuring system

The type of particle for measuring velocity using the PIV method is polystyrene of $50 \mu \mathrm{m}$, $1.016016 \mathrm{~g} / \mathrm{cm}^{2}$. We used ND-Yag Laser, which has an output in trigger mode. The laser sheet created is captured with a high definition CCD camera (Nikon, $1 \mathrm{~K} * 1 \mathrm{~K}, 52 \mathrm{~mm}$ lens), and captured images used for calculations of fluid field by the PIV analysis program, Thinkers. The panorama PIV technique is 
basically analyzing images taken by two cameras installed in the vertical direction.

In this study, the panorama PIV technique is selected in order to obtain nonlinear wave elevation and velocity information along the water depth for a wide range. Since the panorama PIV system's accurate measurement requires information from two separate cameras, two images of a calibration board are taken by two cameras. Then we estimate the relative position of a calibration board and characteristics of the camera lenses. The captured images are converted to one image using a mapping function, and used for calculating 2-D velocity vectors using a cross-correlation PIV method. The basic theory of estimating the velocity field using PIV measurement is measuring the displacement of cast particles in a fluid at a given time segment. For calculating displacement of particles, a particle tracing method by cross-correlation coefficient is used. Some degree of error in the calculation of velocity vectors is expected in this procedure, particularly when the particle information from two consecutive images is not adequate. Therefore it is obvious that sufficient amounts of particle in the fluid will give better results. Fig. 4 shows two images for calibrating two cameras, and Fig. 5 is the resultant images of velocity vectors calculated from a single image, converted from two images using a mapping function.

\section{Results}

The results are mainly comparisons of measurements and theoretical calculations. Fig. 6 and Fig. 7 show wave profiles of the two regular waves that are used for a bichromatic wave. The measured wave height of the regular wave with $\omega_{1}=7.5$ is $0.044 \mathrm{~m}$, and that of the regular wave with $\omega_{2}=6$ is $0.048 \mathrm{~m}$. This measurement proves that a regular wave generated in this wave basin is steady and weakly nonlinear, with a slightly larger amplitude of crest compared to trough.

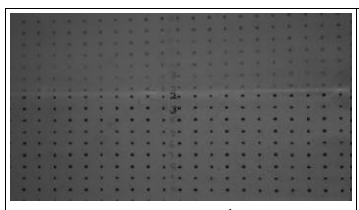

camera 1

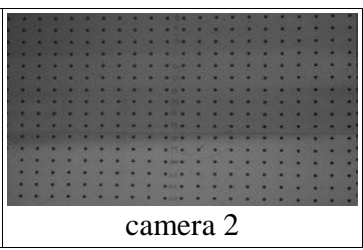

camera 2

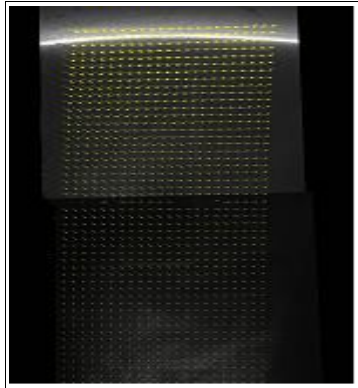

crest

Fig. 5. Measured velocity vector at crest and trough

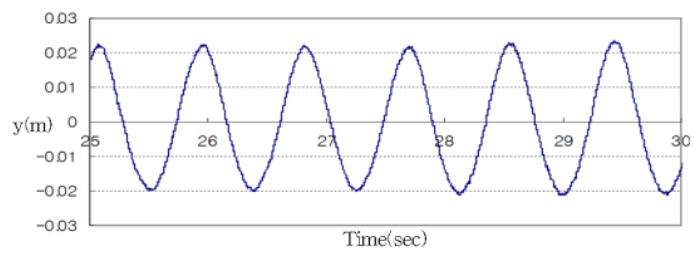

Fig. 6. Measured regular wave $\left(\omega_{1}=7.5, \mathrm{H}=0.044 \mathrm{~m}\right)$

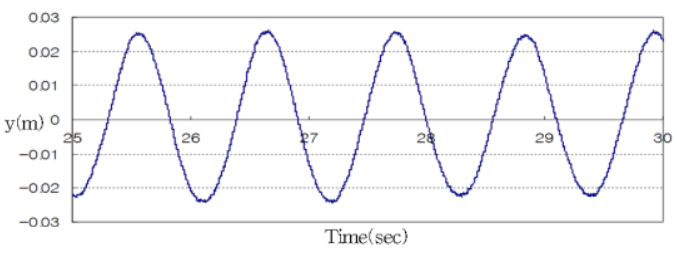

Fig. 7. Measured regular wave $\left(\omega_{2}=6, H=0.048 \mathrm{~m}\right)$

Fig. 8 shows comparison of wave profiles, by measurement and by calculation, of a bichromatic wave composed by phase velocities of two regular waves. The phase difference calculated by the measured wave profile is $0.9 \pi$, and this phase difference is also used for theoretical calculation. A solid line represents the measured profile, the dotted line represents the 1 st order approximation of the theoretical calculation, and the dashed line represents the theoretical calculation approximated up to a 2 nd order term. This figure illustrates the difficulties in simulating completely identical wave profiles along all time instances. It is also known that the effect of nonlinearity is very small when the maximum wave height is smaller than $0.1 \mathrm{~m}$

Fig. 4. Calibration image for camera characteristics 


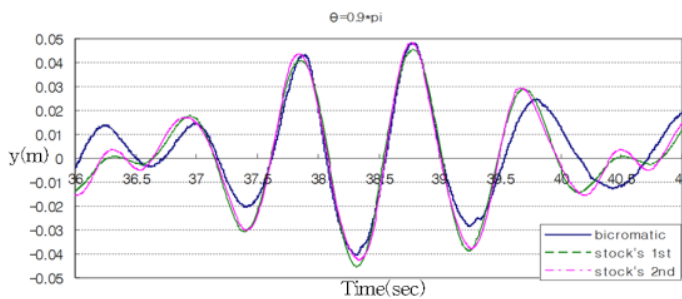

Fig. 8. Wave profile of a bichromatic wave using pure composition

Fig. 9 shows a comparison of wave profiles of bichromatic waves generated by forced composition of two regular waves. The phase difference between two regular waves calculated by measured wave profile is $1.5 \pi$. This method also has difficulties in generating completely identical profiles. When comparing the measured profiles in Fig. 8 and Fig. 9, the forced composition has a sharper crest than the pure composition case, which implies the interaction effect of composition using phase difference causes a slightly blunted crest. However, its effect is very small when the wave height of each regular wave is small, as in this experiment.

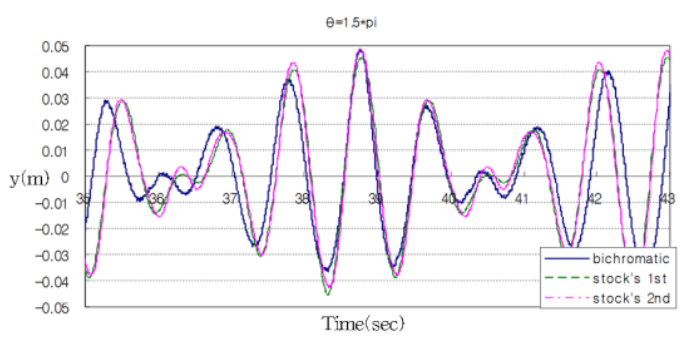

Fig. 9. Wave profile of a bichromatic wave using forced composition

Next, investigations of velocity distribution along water depth from the PIV analysis result will be given at three remarkable locations; two crests and one trough. Fig. 10 shows the locations where the velocity profile will be investigated.

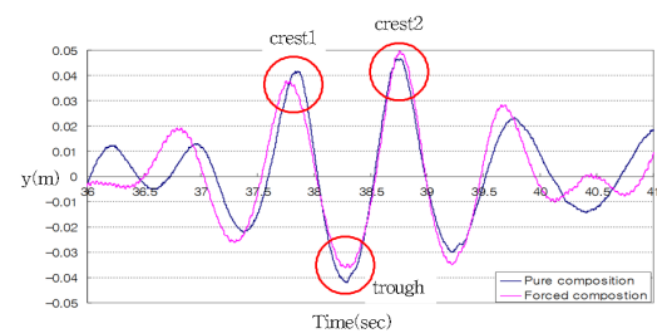

Fig. 10. Selected points for velocity investigation

Fig. 11 and Fig. 12 show the velocity distribution along water depth at the 1st and 2nd crest of a bichromatic wave by pure composition. The symbol " $\diamond$ " means PIV measurement, "+" means 1st order approximation, and " means 2nd order approximation. Good agreement between the PIV measurement and theoretical calculation is shown in these figures. The measurement is slightly small in the lower position of the 1st crest, and its gradient gets larger as it approaches the top of the 1st crest. In Fig. 12, the measured results at the 2nd crest are closer to Stokes 2nd order solution than to the 1 st order solution.

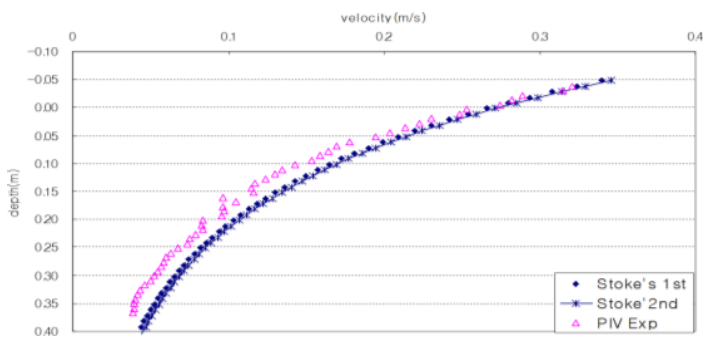

Fig. 11. Velocity profile at 1 st crest of pure composition

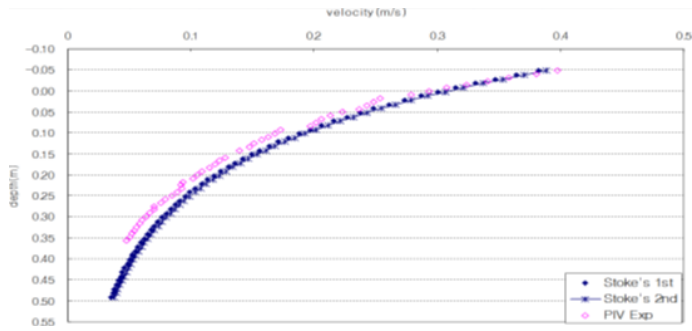

Fig. 12. Velocity profile at 2 nd crest of pure composition

The velocity distribution along water depth at crest 1 and crest 2 of a bichromatic wave generated by forced composition are shown in Fig. 13 and Fig. 14, respectively. The results of forced composition 
are very similar to those of pure composition, and this result confirms that characteristics of a velocity field are not much affected by the methods used to compose a bichromatic wave, whether by pure composition or by forced composition.

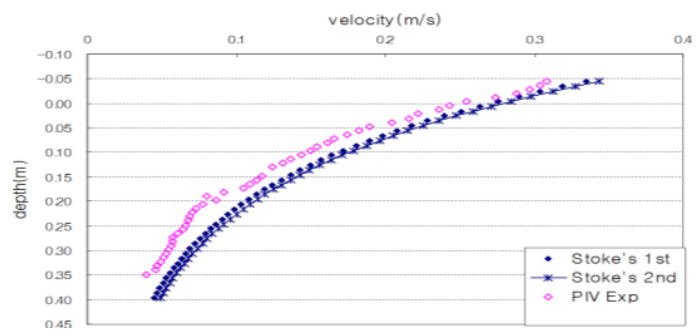

Fig. 13. Velocity profile at 1 st crest of forced composition

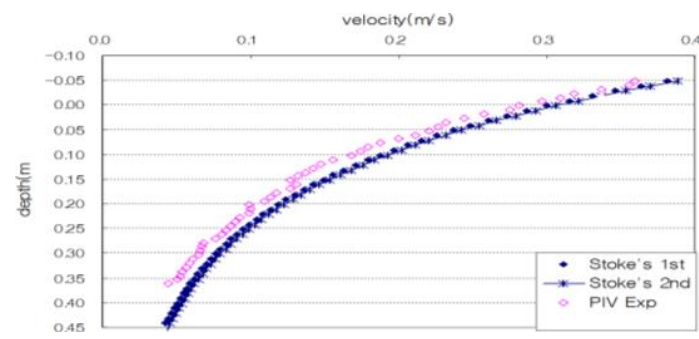

Fig. 14. Velocity profile at 2nd crest of forced composition

A comparison of velocity distribution along water depth at trough by pure composition and by forced composition is presented in Fig. 15. All three sets of results, one measurement and two calculations, agree well.

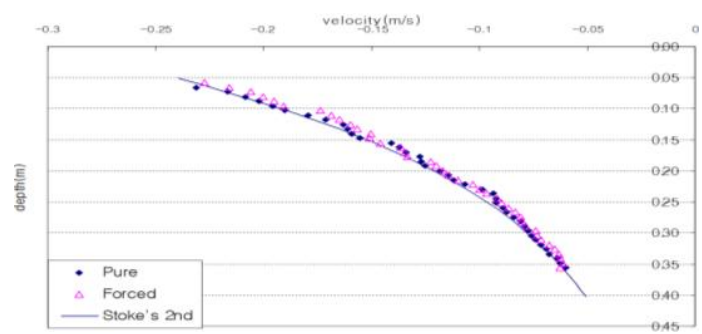

Fig. 15. Velocity profile at trough of pure composition and forced composition

\section{Conclusions}

We generated bichromatic waves by composing two regular waves, and measured their profile and velocity distributions using PIV technique. The measurements were compared with calculations, and the conclusions are:

1) The PIV technique used in this study is applicable to the observation of flow field in waves.

2) The bichromatic wave can be generated by two methods: pure composition of two regular waves, and forced composition by operating a wave maker with synthesized input signal.

3) Measurements and calculations using Stokes theories of bichromatic wave show good agreement.

4) When generating bichromatic waves, the forced composition method does not have much effect on the velocity field of fluid particles.

As stated above, PIV technique is found to be a proper system for describing characteristics of fluid behavior by measuring complex fluid fields. The results from this study need to be further validated through intensive analysis of fluid fields, in comparison with CFD.

\section{Acknowledgements}

This work supported by National Research Laboratory program supported by National Research Foundation (R0A-2008-000-20069-0), Korea.

\section{References}

[1] S.J. Lee, J.G. Choi, J.Y. Sung, T.K. Hwang, and D.H. Doh, Uncertainty Analysis of CrossCorrelation Algorithm based on FFT by PIV Standard Images, Workshop on PIV Techniques and Standards, pp.71-78 (2004).

[2] Chang, K.A. and Liu, PL.F., Velocity, Acceleration and Vorticity under Breaking Waves, Physics of Fluid, Vol. 10, Issue 1, pp.327-329 (1998).

[3] Hudspeth, R.T. and Sulisz, W., Stokes Drift in Two-Dimensional Wave Flumes, Journal of Fluid Mechanics, Vol. 203, pp.209-229 (1991).

[4] Jakobsen, M.L., Dewhirst, T.P. and Greated, C.A., Particle Image Velocimetry for Predictions of Acceleration Fields and Force within Fluid Flow, Journal of Measurement Science and Technology, Vol. 8, pp.1502-1516 (1997).

[5] Jensen, A., Sveen, J.K., Grue, J., Richon, J.B. and Gray, C., Acceleration in Water Waves by 
Extended Particle Imagine Velocimetry, Journal of Experiments in Fluids, Vol. 30, No. 5, pp.500-510 (2001).

[6] Kim, C.H., Randall, R.E., Boo, S.Y. and Krafft, M.J., Kinematics of 2-D Transient Water Using Laser Doppler Anemometry, Journal of Waterway, Port, Coastal, and Ocean Engineering, Vol. 118, Issue 2, pp.147-165 (1992).

[7] Longridge, J.K., Randall, R.E. and Zhang, J., Comparison of Experimental Irregular Water Wave Elevation and Kinematic Data with New Hybrid Wave Model Predictions, Journal of Ocean Engineering, Vol. 23, No. 4, pp.277-307 (1996).

[8] Moubayed, W.L. and Williams, A.N., SecondOrder Bichromatic Wave Produced by a Generic Planar Wavemaker in a TwoDimensional Wave Flume, Journal of Fluids and Structure, Vol. 8, Issue 1, pp.73-92 (1994).
[9] Schäffer, H.A., Second-Order Wavemaker Theory for Irregular Waves, January of Ocean Engineering, Vol. 23, Issue 1, pp.47-88 (1996).

[10]Skjelbreia, J.E., Berek, E., Bolen, J.K., Gudmestad, O.T., Heideman, J., Ohmart, R.D., Spidsøe, N. and Tørum, A. Wave Kinematics in Irregular Waves, International Proceeding of OMAE, Vol. 1, pp.223-228 (1991),

[11]Swan, C., Bashir, T. and Gudmestad, O.T., Nonlinear Inertial Loading. Part 1: Accelerations in Steep 2-D Water Waves, Journal of Fluids and Structures, Vol. 16, No. 3, pp.391416 (2002)

[12]Trulsen, K. and Stansberg, C.T., Spatial Evolution of Water Surface Waves: Numerical Simulation and Experiment of Bichromatic Waves, International Offshore and Polar Engineering Conference, Vol. 3, pp.391-416 (2001). 